\title{
FATORES DE RISCO PARA ULCERAÇÃO NO PÉ DE INDIVÍDUOS COM DIABETES MELLITUS TIPO 2
}

\author{
Elen Ferraz Teston ${ }^{1}$, Juliana de Souza Senteio ${ }^{2}$, Beatriz Maria dos Santos Santiago Ribeiro ${ }^{3}$, Edilaine Maran ${ }^{4}$, \\ Sonia Silva Marcon ${ }^{5}$
}

RESUMO: Objetivou-se analisar os fatores associados ao risco de ulceração do pé em indivíduos com diabetes mellitus tipo 2. Estudo quantitativo, com 71 indivíduos numa Unidade Básica de Saúde de um município do estado do Paraná. Os dados foram coletados em entrevista domiciliar e com exame físico dos pés, no período de julho a setembro de 2016. O pé com risco à ulceração foi identificado em $35,2 \%$ da população estudada. Os fatores de risco às ulcerações mais prevalentes foram o corte inadequado das unhas, a utilização de calçados inadequados, presença de micose, calosidades, rachadura e pele ressecada. O enchimento capilar inadequado, a perda de sensibilidade e o histórico de úlceras estiveram associados ao risco de ulceração. Faz-se mister que os profissionais de saúde reconheçam e valorizem a importância do exame dos pés e do fornecimento de orientações teóricas e práticas, quanto aos cuidados necessários aos pés de indivíduos com diabetes.

DESCRITORES: Diabetes mellitus; Enfermagem; Atenção primária à saúde; Fatores de risco.

\section{RISK FACTORS FOR FOOT ULCERATION IN INDIVIDUALS WITH TYPE 2 DIABETES MELLITUS}

ABSTRACT: The aim of this study was to analyze the factors associated with the risk of foot ulceration in individuals with type 2 diabetes mellitus. Quantitative study, with 71 individuals in a Primary Health Unit of a municipality in the state of Paraná. The data were collected through a home interview and a physical examination of the feet, from July to September 2016. The foot with risk of ulceration was identified in $35.2 \%$ of the studied population. The most prevalent risk factors for ulceration were inadequate nail cutting, use of inadequate footwear, presence of mycosis, callosities and cracked or dry skin. Inadequate capillary filling, loss of sensitivity and history of ulcers were associated with the risk of ulceration. Health professionals should recognize and value the importance of the foot examination and the provision of theoretical and practical guidance regarding foot care for individuals with diabetes.

DESCRIPTORS: Diabetes mellitus; Nursing; Primary healthcare; Risk factors.

\section{FACTORES DE RIESGO PARA ULCERACIÓN EN LOS PIES DE INDIVIDUOS CON DIABETES MELLITUS TIPO 2}

RESUMEN: Fue objetivo del estudio analizar los factores asociados al riesgo de ulceración de los pies de individuos con diabetes mellitus tipo 2. Estudio cuantitativo, con 71 individuos en una Unidad Básica de Salud de un municipio del estado de Paraná. Los datos fueron obtenidos en entrevista domiciliar y con prueba física de los pies, en el periodo de julio a septiembre de 2016. El pie con riesgo de ulceración fue identificado en $35,2 \%$ de la población estudiada. Los factores de riesgo de ulceraciones más prevalentes fueron el corte inadecuado de las uñas, la utilización de zapatos inadecuados, presencia de micosis, callosidades, rajadura y piel seca. El llenado capilar inadecuado, la pérdida de sensibilidad y el histórico de úlceras estuvieron asociados al riesgo de ulceración. Se concluye que es esencial que los profesionales de salud reconozcan y valoren la importancia de la prueba de los pies y de orientaciones teóricas y prácticas acerca de los cuidados necesarios a los pies de individuos con diabetes.

DESCRIPTORES: Diabetes mellitus; Enfermería; Atención básica a la salud; Factores de riesgo.

${ }^{1}$ Enfermeira. Doutora em Enfermagem. Docente da Universidade Estadual do Paraná. Paranavaí, PR, Brasil.

${ }^{2}$ Discente de Enfermagem. Universidade Estadual do Paraná. Paranavaí, PR, Brasil.

${ }^{3}$ Discente de Enfermagem. Faculdade de Apucarana. Paranavaí, PR, Brasil.

${ }^{4}$ Enfermeira. Mestre em Enfermagem. Docente da Universidade Estadual do Paraná. Paranavaí, PR, Brasil.

${ }^{5}$ Enfermeira. Doutora em Filosofia da Enfermagem. Docente da Universidade Estadual de Maringá. Maringá, PR, Brasil.

Autor Correspondente:

Recebido: 29/03/2017

Elen Ferraz Teston

Finalizado: 20/10/2017

Universidade Estadual do Paraná

Av. Gabriel Esperidião, S/N - 87703-000 - Paranavaí, PR, Brasil

E-mail: ferrazteston@gmail.com 


\section{INTRODUÇÃO}

O Diabetes Mellitus (DM) é caracterizado por elevados níveis de glicose no sangue decorrente de falha na produção e/ou incapacidade da insulina em desempenhar adequadamente as suas funções ${ }^{(1)}$. Sua complexidade associada à elevada incidência e prevalência concorrem para que essa doença seja considerada um importante problema de saúde pública ${ }^{(2)}$.

No ano de 2014 cerca de 347 milhões de pessoas em todo o mundo possuíam o diagnóstico de DM, o que equivale a $6 \%$ da população mundial ${ }^{(3)}$. O Brasil ocupa a quinta posição no ranking das nações com maior número de indivíduos acometidos ${ }^{(4)}$.

O DM tipo 2 (DM2) é o mais frequente e representa de $90 \%$ a 95\% dos casos. Sua ocorrência está associada a comportamentos, como a alimentação não saudável, sedentarismo, fatores de risco como a obesidade e hipertensão, além de fatores genéticos ${ }^{(1)}$.

O descontrole glicêmico ou o diagnóstico tardio aumentam a chance de complicações, tais como doenças cardiovasculares, retinopatia, nefropatia e pé diabético ${ }^{(1)}$. O pé diabético ocorre quando uma área lesionada ou infeccionada nos pés desenvolve úlcera ${ }^{(5)}$. Acomete cerca de $15 \%$ dos pacientes com DM ao longo da vida e é responsável por mais de $60 \%$ das amputações não traumáticas ${ }^{(1-2)}$, o que consequentemente gera ampla repercussão social e econômica ${ }^{(6)}$.

Dados referentes às pessoas inscritas no sistema de seguros de saúde, gerido pelos Estados Unidos da América, mostraram que $24,4 \%$ do total gasto com a saúde no país foi dispendido no cuidado a indivíduos com DM2 que apresentavam pé diabético ${ }^{(7)}$.

Destarte, conhecer os fatores associados que aumentam o risco para o desenvolvimento de pé diabético auxilia no planejamento de ações a serem desenvolvidas pela equipe de enfermagem para prevenção dessa complicação. Os pés de indivíduos com DM requerem cuidados contínuos e sua inspeção deve ser incluída na rotina de atendimento a esses indivíduos. Atinente a isso, o presente estudo teve como objetivo analisar os fatores associados ao risco de ulceração do pé em indivíduos com DM2.

\section{MÉTODO}

Estudo quantitativo, transversal, realizado em uma Unidade Básica de Saúde (UBS) de um município de médio porte, situado na região noroeste do estado do Paraná. O município conta com 15 UBS e cobertura populacional de $100 \%$ pelas Equipes de Estratégia Saúde da Família (ESF). Até o último quadrimestre de 2015, 2.286 indivíduos estavam cadastrados nas UBS do município com diagnóstico de DM. A UBS selecionada, por conveniência, conta com duas equipes da ESF, sendo uma delas o campo de atuação do estágio supervisionado obrigatório do $4^{\circ}$ ano da graduação em enfermagem, ocasião da pesquisa.

Os participantes do estudo foram os indivíduos com DM2 cadastrados na referida UBS. No ano de 2016 estavam cadastrados em uma das equipes da ESF, cenário do estudo, 106 indivíduos com 40 anos ou mais, com diagnóstico de DM2.

Para o cálculo amostral utilizou-se a técnica de amostragem probabilística considerando o erro de estimativa de $5 \%$, nível de confiança de $95 \%$ e prevalência de $50 \%$, para obter maior variabilidade do evento estudado, acrescida de $10 \%$ para possíveis perdas ou exclusões. Com estes parâmetros, obtevese número amostral de 84 indivíduos. Procedeu-se ao sorteio aleatório dos participantes, a fim de que os dados levantados fossem representativos do todo, a partir da lista com o nome e endereço dos indivíduos com DM2 fornecida pela equipe da ESF.

Os dados foram coletados no período de julho a setembro de 2016, por meio de entrevista domiciliar e exame físico dos pés. Para registro das informações referentes à avaliação dos membros inferiores, incluindo condição dermatológica, circulatória, neuropática e de cuidado com os pés, foram utilizadas as variáveis constantes no caderno de Atenção Básica ${ }^{(4)}$. 
Os critérios de inclusão foram: indivíduos de ambos os sexos, com idade igual ou superior a 40 anos. Os critérios de exclusão foram apresentar sequelas que prejudicassem a comunicação e diagnóstico de alguma doença mental.

$\mathrm{Na}$ avaliação dos membros inferiores foram consideradas as seguintes variáveis: 1. Práticas de cuidado com os pés (após o banho seca entre os dedos dos pés; avalia os pés; faz escalda pés; caminha descalço; calçados utilizados diariamente); 2. Exame dos pés (corte das unhas; calçados utilizados no momento da entrevista; higiene) - quanto ao tipo de calçado utilizado, foi considerado adequado o modelo fechado, com um centímetro a mais em toda a extensão interna do sapato, para não ficar apertado e nem largo, confeccionado em material tipo couro macio ou lonalalgodão ${ }^{(8)}$; 3 . Aspectos dermatológicos (presença de micose em unha e interdigital, calosidade, rachaduras nos pés, pele ressecada); 4. Presença de neuropatia identificada com o teste do monofilamento Semmes-Weinstein de $10 \mathrm{~g}$ gramas $^{(4)}$; 5 . Presença de deformidades (hálux valgo, dedos em garra, dedos em martelo e proeminências ósseas); e 6. Alteração vascular (palpação dos pulsos tibiosos e pediais posteriores).

A classificação do risco de ulceração foi realizada conforme Caderno de atenção básica: Grau 0: Neuropatia ausente (sensibilidade preservada); Grau 1: Neuropatia presente (alteração de sensibilidade); Grau 2: Neuropatia presente, sinais de doença vascular periférica elou deformidade nos pés; Grau 3: amputação prévia(4).

Para análise, os dados foram primeiramente lançados em planilhas do programa Microsoft Office Excel 2010 e posteriormente transferidos para o programa estatístico IBM SPSS versão 20. Para identificação de associações entre as variáveis, foi utilizado teste de Qui-quadrado com correção Yates. Em todos os testes foi considerado o nível de significância de 5\%.

A pesquisa foi aprovada em junho de 2016 pelo Comitê de Ética da Universidade Estadual de Maringá, sob parecer $\mathrm{n}^{\mathrm{0}} 1.606 .073$.

\section{RESULTADOS}

Participaram do estudo 71 indivíduos, pois 13 não foram encontrados após três visitas domiciliares. Vale salientar que a perda não impacta nos resultados, uma vez que durante a realização do cálculo amostral considerou-se a previsão de possíveis perdas. Destes, $62 \%$ (44) eram do sexo feminino, com média de idade de 60 anos (desvio padrão = 10,2 anos). Com relação à cor, 71,8\% (51) se autorreferiram brancos, e 69,6\% (49) possuíam companheiro. Quanto ao nível de escolaridade houve predominância de indivíduos com menos de seis anos de estudo 67,7\% (48). Destaca-se que 67,6\% (48) possuíam o diagnóstico de DM2 há 10 anos ou mais (tempo médio de diagnóstico de 11 anos) e 26,8\% (19) faziam uso de insulina.

No exame clínico dos pés, $81,7 \%$ (58) dos participantes apresentaram boas condições de higiene. O corte das unhas esteve inadequado em $36,6 \%$ (26) e parcialmente adequado em $29,6 \%$ (21). O hábito de secar diariamente os espaços interdigitais dos pés foi referido por $59 \%$ (41).

Segundo o relato dos participantes, 43,7\% (31) auto avaliavam os pés diariamente e 32,4\% (23) às vezes. A prática de escalda pés foi negada por 59\% (41). Não ter o hábito de andar descalço ou andar às vezes foi encontrado, respectivamente em 63,4\% (45) e 29,6\% (21) dos entrevistados. Dos indivíduos examinados, $71,8 \%$ (51) se apresentaram com calçado inadequado no momento da entrevista.

Não houve associação estatisticamente significativa entre as ações de autocuidado com os pés e a classificação de risco à ulceração, conforme se verifica na Tabela 1. 
Tabela 1 - Distribuição do autocuidadocom os pés, de acordo com a classificação de risco de ulceração em pés de diabéticos tipo 2. Paranavaí, PR, Brasil, 2016

\begin{tabular}{|c|c|c|c|c|}
\hline \multirow[t]{2}{*}{ Autocuidado } & \multicolumn{4}{|c|}{$(\%)$ Risco de Ulceração $(n=71)$} \\
\hline & Grau 0 & Grau 1 & Grau 2 & Valor $p$ \\
\hline \multicolumn{5}{|l|}{ Higiene } \\
\hline Adequado & 63,8 & 36,2 & - & \multirow[t]{2}{*}{0,08} \\
\hline Inadequado & 69,2 & 23,1 & 7,7 & \\
\hline \multicolumn{5}{|l|}{ Seca entre dedos } \\
\hline Nunca & 54,5 & 45,5 & - & \multirow[t]{3}{*}{0,506} \\
\hline Às vezes & 85,7 & 14,3 & - & \\
\hline Diariamente & 66,7 & 31 & 2,3 & \\
\hline \multicolumn{5}{|l|}{ Avalia os pés } \\
\hline Nunca & 52,9 & 47,1 & - & \multirow[t]{3}{*}{0,566} \\
\hline Às vezes & 69,6 & 30,4 & - & \\
\hline Diariamente & 67,7 & 29 & 3,2 & \\
\hline \multicolumn{5}{|l|}{ Faz escalda pés } \\
\hline Nunca & 64,3 & 33,3 & 2,4 & \multirow[t]{3}{*}{0,763} \\
\hline Às vezes & 63 & 37 & - & \\
\hline Diariamente & 100 & - & - & \\
\hline \multicolumn{5}{|l|}{ Caminha descalço } \\
\hline Nunca & 57,8 & 40 & 2,2 & \multirow[t]{3}{*}{0,152} \\
\hline Às vezes & 85,7 & 14,3 & - & \\
\hline Diariamente & 40 & 60 & - & \\
\hline \multicolumn{5}{|l|}{ Corte das unhas } \\
\hline Adequado & 70,8 & 29,2 & - & \multirow[t]{3}{*}{0,663} \\
\hline Parcial Adequado & 66,7 & 33,3 & - & \\
\hline Inadequado & 57,7 & 38,5 & 3,8 & \\
\hline \multicolumn{5}{|l|}{ Calçado utilizado } \\
\hline Adequado & 76,2 & 19 & 4,8 & \multirow[t]{2}{*}{0,086} \\
\hline Inadequado & 60 & 40 & - & \\
\hline
\end{tabular}

Com relação às alterações dermatológicas, 52,1\% (37) dos indivíduos apresentaram onicomicose, enquanto que $18,3 \%$ (13) apresentaram micose nos espaços interdigitais. A presença de calos foi verificada em $56,3 \%$ (40), rachaduras em $60,6 \%$ (43) e 78,9\% (56) possuíam xerose. Entretanto, essas alterações não tiveram associação estatisticamente significativa com o risco à ulceração, conforme se observa na Tabela 2.

Tabela 2 - Distribuição das alterações dermatológicas, de acordo com a classificação de risco de ulceração em pés de diabéticos tipo 2. Paranavaí, PR, Brasil, 2016 (continua)

\begin{tabular}{lcccc} 
Alterações Dermatológicas & \multicolumn{3}{c}{$\mathbf{( \% )}$} & Risco de Ulceração $(\mathbf{n}=\mathbf{7 1})$ \\
\cline { 2 - 4 } & Grau 0 & Grau 1 & Grau 2 & Valor $\mathbf{p}$ \\
\hline Onicomicose & & & & \\
\hline Sim & 62,2 & 35,1 & 2,7 & \multirow{2}{*}{0,594} \\
\hline Não & 67,6 & 32,4 & - & \\
\hline Micose interdigital & & & & \\
\hline Sim & 53,8 & 38,5 & 7,7 & \multirow{2}{*}{0,088} \\
\hline Não & 67,2 & 32,8 & - & \\
\hline \hline
\end{tabular}




\begin{tabular}{|c|c|c|c|c|}
\hline \multicolumn{5}{|c|}{ Calosidades } \\
\hline Sim & 65 & 32,5 & 2,5 & \multirow[t]{2}{*}{0,663} \\
\hline Não & 64,5 & 35,5 & - & \\
\hline \multicolumn{5}{|c|}{ Rachaduras } \\
\hline Sim & 55,8 & 41,9 & 2,3 & \multirow[t]{2}{*}{0,129} \\
\hline Não & 78,6 & 21,4 & - & \\
\hline \multicolumn{5}{|c|}{ Xerose } \\
\hline Sim & 60,7 & 37,5 & 1,8 & \multirow[t]{2}{*}{0,364} \\
\hline Não & 80 & 20 & - & \\
\hline
\end{tabular}

Quanto às alterações vasculares, verificou-se que o pulso pedioso estava diminuído em 23,9\% (17) e ausente em 8,5\% (6). O pulso tibial posterior estava diminuído em 38\% (27) e ausente em 22,5\% (16). A sensibilidade esteve ausente em $22 \%$ (15) dos indivíduos e $25 \%$ (18) apresentaram preenchimento capilar inadequado.

O enchimento capilar inadequado, a perda de sensibilidade e o histórico de úlcera estiveram associados estatisticamente ao risco de ulceração.

Tabela 3 - Distribuição das alterações vasculares, ortopédicas e neurológica, de acordo com a classificação de risco de ulceração em pés de diabéticos tipo 2. Paranavaí, PR, Brasil, 2016 (continua)

\begin{tabular}{|c|c|c|c|c|}
\hline \multirow[t]{2}{*}{ Características } & \multicolumn{4}{|c|}{ (\%) Risco de Ulceração $(\mathrm{n}=71)$} \\
\hline & Grau 0 & Grau 1 & Grau 2 & Valor $p$ \\
\hline \multicolumn{5}{|l|}{ Pulso Pedioso D } \\
\hline Palpável & 71,7 & 28,3 & - & \multirow[t]{3}{*}{0,002} \\
\hline Diminuído & 46,2 & 53,8 & - & \\
\hline Não palpável & 40 & 40 & 20 & \\
\hline \multicolumn{5}{|l|}{ Pulso Pedioso E } \\
\hline Palpável & 72,2 & 27,8 & - & \multirow[t]{3}{*}{0,004} \\
\hline Diminuído & 45,5 & 54,5 & - & \\
\hline Não palpável & 33,3 & 50 & 16,7 & \\
\hline \multicolumn{5}{|l|}{ Pulso Tibial D } \\
\hline Palpável & 79,3 & 20,7 & - & \multirow[t]{3}{*}{0,096} \\
\hline Diminuído & 55,6 & 44,4 & - & \\
\hline Não palpável & 53,3 & 40 & 6,7 & \\
\hline \multicolumn{5}{|l|}{ Pulso Tibial E } \\
\hline Palpável & 82,4 & 17,6 & - & \multirow[t]{3}{*}{0,010} \\
\hline Diminuído & 52 & 48 & - & \\
\hline Não palpável & 41,7 & 50 & 8,3 & \\
\hline \multicolumn{5}{|c|}{ Enchimento Capilar D } \\
\hline Adequado & 72,2 & 27,8 & - & \multirow[t]{2}{*}{0,023} \\
\hline Inadequado & 41,2 & 52,9 & 5,9 & \\
\hline \multicolumn{5}{|c|}{ Enchimento Capilar E } \\
\hline Adequado & 34,3 & 17,9 & - & \multirow[t]{2}{*}{0,012} \\
\hline Inadequado & 38,9 & 55,6 & 5,6 & \\
\hline \multicolumn{5}{|l|}{ Deformidades D } \\
\hline Sim & 66,7 & 33,3 & - & \multirow[t]{2}{*}{0,53} \\
\hline Não & 62,5 & 34,4 & 3,1 & \\
\hline
\end{tabular}




\begin{tabular}{|c|c|c|c|c|}
\hline \multicolumn{5}{|c|}{ Deformidades E } \\
\hline Sim & 64,9 & 35,1 & - & \multirow[t]{2}{*}{0,569} \\
\hline Não & 64,7 & 32,4 & 2,9 & \\
\hline \multicolumn{5}{|c|}{ Perda de Sensibilidade D } \\
\hline Sim & 0 & 95 & 5 & \multirow[t]{2}{*}{$\mathbf{0}$} \\
\hline Não & 90,2 & 9,8 & - & \\
\hline \multicolumn{5}{|c|}{ Perda de Sensibilidade E } \\
\hline Sim & 0 & 94,7 & 5,3 & \multirow[t]{2}{*}{$\mathbf{0}$} \\
\hline Não & 88,5 & 11,5 & - & \\
\hline \multicolumn{5}{|c|}{ História de Úlcera D } \\
\hline Sim & 20 & 70 & 10 & \multirow[t]{2}{*}{$\mathbf{0 , 0 0 1}$} \\
\hline Não & 72,1 & 27,9 & - & \\
\hline \multicolumn{5}{|c|}{ História de Úlcera E } \\
\hline Sim & 50 & 50 & 0 & \multirow[t]{2}{*}{0,879} \\
\hline Não & 65,2 & 33,3 & 1,4 & \\
\hline
\end{tabular}

\section{- DISCUSSÃO}

Os resultados encontrados corroboram estudo que demonstra o quanto pessoas com baixa escolaridade podem apresentar risco maior para o desenvolvimento do pé diabético, visto que o baixo nível de instrução interfere na compreensão e adesão ao tratamento proposto para controle do diabetes e suas complicações ${ }^{(9)}$. Além da escolaridade, o tempo de diagnóstico também constitui fator indicativo de gravidade para o aparecimento de úlceras em membros inferiores ${ }^{(10)}$.

Nesse sentido, considerando que a maioria dos participantes do presente estudo apresentou tempo de diagnóstico maior que 10 anos, destaca-se a importância de aumentar as ações de vigilância, por parte dos profissionais de saúde, com relação à investigação dos fatores de risco modificáveis para ulceração dos pés, com vistas ao desenvolvimento de intervenções precoces.

Uma das principais consequências inicialmente do diabetes mellitus é o pé diabético, caracterizado pelo surgimento de úlceras nos pés ${ }^{(4)}$. Esta complicação traz algumas consequências além da simples limitação do membro, tais como diminuição da qualidade de vida, elevados custos com hospitalizações repetitivas, reabilitação por longos períodos e mortalidade prematura ${ }^{(11)}$. O índice de lesões nos pés e consequentemente ulcerações pode ser reduzido mediante o conhecimento prévio dos fatores de risco, a adoção de medidas preventivas e cuidados adequados. Dentre as medidas preventivas e cuidados adequados com os pés, condições de boa higiene estiveram presentes com um percentual expressivo neste estudo, o que denota preocupação dos indivíduos em manter os membros saudáveis, livres de lesões e úlceras.

Embora 59\% dos participantes deste estudo tenham referido que secam diariamente os pés, é importante lembrar que o hábito de não secar os pés entre os dedos pode propiciar a proliferação de micro-organismos e ocasionar fissuras e doenças na pele ${ }^{(4)}$. Hábitos como corte inadequado das unhas, andar descalços e uso de sapatos inadequados, prevalentes entre os participantes do presente estudo, podem desencadear risco à integridade da pele dos pés, especialmente para os diabéticos, visto que estes indivíduos possuem sensibilidade diminuída ${ }^{(12)}$.

Em um estudo sobre conhecimento, atitudes e práticas de medidas preventivas sobre pé diabético, demonstrou-se que as mulheres realizam hidratação e massagem nas extremidades inferiores e praticam o orientado para a prevenção do pé diabético, já os homens, por questões culturais, atribuem os cuidados ao sexo feminino e não adotam as recomendaçõe ${ }^{s(13)}$. Nesse contexto, fatores como comportamentos relacionados ao sexo devem ser considerados durante as orientações realizadas pelos profissionais de saúde. 
Para a obtenção de bons resultados na adesão ao autocuidado, é necessário planejamento de ações preventivas e que estas abordem a adoção de cuidados específicos com os pés, exame diário, hidratação da pele, corte adequado das unhas e não remoção de calosidades, entre outros. Os profissionais de saúde, em especial os da Estratégia Saúde da Família, são responsáveis pelo desenvolvimento de ações promoção, proteção e recuperação da saúde. Portanto, a identificação dos principais fatores de risco para o desenvolvimento de ulceração é essencial para o planejamento de ações de prevenção e retardo de complicações nos pés, favorecendo a qualidade de vida de pessoas com diabetes ${ }^{(13)}$.

Apesar da inobservância da associação entre autocuidado e o grau de risco para ulceração neste estudo, é importante considerar estes aspectos no cotidiano das pessoas com DM. Pesquisa em um Centro de Saúde de Teresina-PI constatou que, dos 331 indivíduos com DM2, 178 referiram não ter recebido orientação do enfermeiro quanto à importância do exame dos pés e a secagem dos espaços interdigitais, e ainda, 66,5\% (220) negaram ter recebido orientação quanto à inspeção dos sapatos antes de calçá-los ${ }^{(14)}$.

Estes dados permitem inferir que os profissionais precisam persistir em seu papel fundamental à promoção da saúde. Destarte, a população em geral e em especial as pessoas com condição crônica, tal como o DM2, necessitam de intervenções contínuas que favoreçam a qualidade de vida e a prevenção de complicações.

O autocuidado diz respeito aos comportamentos adotados pelos indivíduos em seu cotidiano. Pessoas com menores condições financeiras e de escolaridade têm maior propensão à descompensação glicêmica e elevados índices de complicações do DM, o que aumenta a taxa de mortalidade desta população ${ }^{(15)}$. Mediante esse cenário e a dificuldade que esses indivíduos têm de conseguir assistência efetiva, é necessária a disseminação da importância do autocuidado e os benefícios adquiridos a partir dele.

Além disso, as dificuldades financeiras e de escolaridade também influenciam na aquisição de calçados adequados, como foi verificado em $71,8 \%$ dos participantes. Neste sentido, ressaltamos a importância de os profissionais oferecerem à população possibilidades de adaptações que favoreçam a adoção de práticas de cuidado no cotidiano, considerando a realidade vivenciada.

As alterações dermatológicas, por sua vez, são consequências de autocuidado ineficaz e que trazem alguns prejuízos para o indivíduo com DM. No presente estudo evidenciou-se um número significativo de indivíduos com xerose (pele ressecada) e onicomicose (micose nas unhas). Resultado análogo foi encontrado em estudo ${ }^{(16)}$ realizado em um Hospital Universitário junto a 212 indivíduos com DM que apresentaram algumas afecções nos pés, entre elas a xerose $(55,2 \%)$ e a onicomicose $(43,9 \%)$, o que demonstra o quanto o DM prejudica a integridade da pele se não houver os devidos cuidados. Daí a importância dos profissionais de saúde orientar a população quanto às práticas de inspeção dos pés, promovendo ações dinâmicas capazes de transmitir informações de forma acessível e que envolvam a demonstração e supervisão de cuidados específicos.

A doença vascular periférica é considerada um dos fatores mais importantes para o desenvolvimento de ulceração e destruição de tecidos profundos ${ }^{(17)}$. Neste estudo, verificou-se percentual importante de indivíduos com diminuição e ausência de pulso tibial e pedioso, semelhante a pesquisa realizada em Parnaíba-PI, a qual identificou elevada prevalência da diminuição do pulso pedioso nos membros direito e esquerdo (51,3\% e 53,9\% respectivamente) e diminuição do pulso tibial esquerdo em 56,5\% ${ }^{(18)}$.

Estes dados e os obtidos no presente estudo demonstram que os indivíduos com DM2 apresentam deficiência do fluxo sanguíneo nos membros inferiores. Essa deficiência deve ser identificada e valorizada durante o acompanhamento pelos profissionais de saúde. Vale salientar que, embora esse fator não possa ser modificado, existem cuidados que evitam consequências maiores como, por exemplo, a prática de manter os membros elevados por pequenos períodos durante o dia.

A neuropatia diabética é uma consequência do DM e está associada à perda da sensibilidade cutânea em média em $80 \%$ dos casos. Esses dois fatores associados podem levar o indivíduo ao desenvolvimento de uma ulceração nos pés ${ }^{(4)}$. Embora pouco prevalentes no presente estudo, tanto a perda da sensibilidade (22\%) quanto o enchimento capilar inadequado (25\%) merecem atenção no processo de monitoramento e acompanhamento desses indivíduos, uma vez que se observou significativa estatística entre perda de sensibilidade e risco de ulceração. 
Essa situação mostra que quanto maior o prejuízo da sensibilidade, maior chance do membro sofrer um trauma sem que o indivíduo perceba, bem como os riscos para que uma lesão evolua para ulceração. Vale ressaltar que, além da lesão no membro, essa condição também desgasta emocionalmente o indivíduo.

As alterações glicêmicas frequentes possuem maiores chances de complicações do DM, entre elas a doença vascular periférica, que pode ser decorrente de um trauma nos pés que, quando associado à descompensação glicêmica, pode desenvolver o pé diabético ${ }^{(19)}$. Isto por si só já indica a necessidade de estas pessoas terem acompanhamento multiprofissional, para que sejam adequadamente instrumentalizadas em relação ao autocuidado e gerenciamento de sua vida.

Entre as complicações crônicas do DM, a ulceração e a amputação dos pés são as mais graves e de grande impacto socioeconômico(8). No presente estudo, foi observada associação significativa entre histórico de úlcera e risco para ulceração grau 1. Ou seja, o indivíduo com úlcera prévia apresenta maior chance de novos casos de úlceras e maior probabilidade de amputação de membro, o que indica que estes indivíduos necessitam de cuidado redobrado e melhor manejo da doença.

Destaca-se como limitação o fato de que este estudo se concentrou nos fatores associados ao risco de ulceração do pé, em indivíduos com DM2, específica de uma UBS. Assim, estes resultados revelam a problemática de uma realidade local e, portanto, em virtude da amostra e realização em uma única instituição, os dados não são generalizáveis. Este estudo sugere que sejam aplicadas novas pesquisas em outras UBS e com isto fazer uma avaliação mais ampla da problemática.

\section{CONSIDERAÇÕES FINAIS}

Os resultados deste estudo permitem inferir que os profissionais de saúde necessitam engajar-se efetivamente na assistência e orientação de indivíduos com DM, uma vez que parcela considerável das pessoas cadastradas nesta UBS apresenta pé de risco à ulceração e fatores de risco modificáveis, como o corte das unhas e a utilização de calçados inadequados, presença de micose, calos, rachaduras e pele ressecada.

Torna-se oportuno articular que a precarização dos serviços de saúde e a cultura dos indivíduos, em especial a dos homens, implicam na reorganização do planejamento da assistência pela ESF e na efetividade de práticas de educação em saúde para doenças crônicas.

Espera-se que os indivíduos com DM realizem os cuidados específicos apreendidos e sejam acompanhados continuamente pelos enfermeiros e profissionais de saúde, evitando e minimizando as chances de desenvolvimento de ulceração, que interfere nas condições e qualidade de vida dessa população.

\section{REFERÊNCIAS}

1. Sánchez-Zamora YI, Rodriguez-Sosa M. The role of MIF in type 1 and type 2 Diabetes Mellitus. J Diabetes Res. [Internet] 2014;(804519) [acesso em 05 dez 2016]. Disponível: http://dx.doi.org/10.1155/2014/804519.

2. Sargis RM. The hijacking of cellular signaling and the diabetes epidemic: mechanisms of environmental disruption of insulin action and glucose homeostasis. Diabetes Metab J. [Internet] 2014;38(1) [acesso em 10 nov 2016]. Disponível: http://dx.doi.org/10.4093/dmj.2014.38.1.13.

3. World Health Organization (WHO). WHOGlobal action plan for the prevention and control of noncommunicable disease 2013-2020. [Internet] Geneva: World Health Organization; 2013 [acesso em 10 nov 2016]. Disponível: http://www.who.int/nmh/events/ncd_action_plan/en/.

4. Ministério da Saúde (BR). Cadernos de Atenção Básica. Estratégias para o cuidado da pessoa com doença crônica. Diabetes Mellitus. [Internet] Brasília (DF): Ministério da Saúde; 2013 [acesso em 20 out 2016]. Disponível: http://bvsms.saude.gov.br/bvs/publicacoes/estrategias_cuidado_pessoa_diabetes_mellitus_cab36.pdf.

5. Ministério da Saúde (BR). Manual do pé diabético 2016: Estratégia para o cuidado da pessoa com doença 
crônica. [Internet] Brasília: Ministério da Saúde; 2016 [acesso em 15 out 2016]. Disponível: http://189.28.128.100/ dab/docs/portaldab/publicacoes/manual_do_pe_diabetico.pdf.

6. de Almeida SA, Silveira MM, Santo PFE, Pereira RC, Salomé GM. Avaliação da qualidade de vida em pacientes com diabetes mellitus e pé ulcerado. Rev. Bras. Cir. Plást. [Internet] 2013;28(1) [acesso em 12 out 2016]. Disponível: http://dx.doi.org/10.1590/S1983-51752013000100024.

7. Sargen MR, Hoffstad O, Margolis DJ. Geographic variation in Medicare spending and mortality for diabetic patients with foot ulcers and amputations. J Diabetes Complications. [Internet] 2013;27(2) [acesso em 12 out 2016]. Disponível: http://dx.doi.org/10.1016/j.jdiacomp.2012.09.003.

8. International Working Group on the Diabetic Foot (IWGDF). International Consensus on the Diabetic Foot. [DVD]. [Internet] Amsterdam: IWGDF; 2011 [acesso em 15 out 2016]. Disponíevl: http://www.iwgdf.org/.

9. Brito CKD, Nottingham IC, Victor JF, Feitoza SMS, da Silva MG, do Amaral HEG. Úlcera venosa: avaliação clínica, orientações e cuidados com o curativo. Rev Rene. [Internet] 2013;14(3) [acesso em 06 ago 2016]. Disponível: http://www.revistarene.ufc.br/revista/index.php/revista/article/viewFile/689/pdf.

10. Targino IG, Souza JSO, dos Santos NMG, Davim RMB, da Silva RAR. Fatores relacionados ao desenvolvimento de úlceras em pacientes com Diabetes Mellitus. Rev Fund Care Online. [Internet] 2016;8(4) [acesso em 02 nov 2016]. Disponível: http://dx.doi.org/10.9789/2175-5361.2016.v8i4.4929-4934.

11. Ferreira V, Martins J, Loureiro L, Loureiro T, Borges L, Silveira D, et al. Consulta multidisciplinar do pé diabético - avaliação dos fatores de mau prognóstico. Angiol Cir Vasc. [Internet] 2014;10(3) [acesso em 02 nov 2016]. Disponível: http://www.scielo.mec.pt/scielo.php?script=sci_arttext\&pid=S1646-706X2014000300005\&Ing $=$ en\&nrm=i\&tlng=pt.

12. Silva PL, Rezende MP, Ferreira LA, Dias FA, Helmo FR, Silveira FCO. Cuidados com os pés: o conhecimento de indivíduos com diabetes mellitus cadastrados no programa saúde da familia. Enfermería Global. [Internet] 2015;(37) [acesso em 05 nov 2016]. Disponível: http://scielo.isciii.es/pdf/eg/v14n37/pt_clinica3.pdf.

13. Policarpo NS, Moura JRA, de Melo Júnior EB, de Almeida PC, de Macêdo SF, da Silva ARV. Conhecimento, atitudes e práticas de medidas preventivas sobre pé diabético. Rev. Gaúcha Enferm. [Internet] 2014;35(3) [acesso em 05 nov 2016]. Disponível: http://seer.ufrgs.br/index.php/RevistaGauchadeEnfermagem/article/view/45187.

14. Rezende Neta DS, da Silva ARV, da Silva GRF. Adesão das pessoas com diabetes mellitus ao autocuidado com os pés. Rev. bras. enferm. [Internet] 2015;68(1) [acesso em 19 jan 2017]. Disponível: http://dx.doi.org/10.1590/00347167.2015680115p.

15. Pereira MG. Beyond life style interventions in Type 2 Diabetes. Rev. Latino-Am. Enfermagem. [Internet] 2016;24(2765) [acesso em 19 jan 2017]. Disponível: http://dx.doi.org/10.1590/1518-8345.0000.2765.

16. de Lima IG, Costa JFL, Oliveira AF, Borges Junior JN, Peixoto AS, Pancieri MS, et al. Educar para prevenir: A importância da informação no cuidado do pé diabético. Revista Conexão UEPG. [Internet] 2017;13(1) [acesso em 19 jan 2017]. Disponível: http://dx.doi.org/10.5212/Rev.Conexao.v.13.i1.0015.

17. Bakker K, Schaper NC, International Working Group on Diabetic Foot Editorial Board. The development of global consensus guidelines on the management and prevention of the diabetic foot 2011. Diabetes Metab Res Rev. [Internet] 2012;28(Suppl 1) [acesso em 04 abr 2017]. Disponível: http://dx.doi.org/10.1002/dmrr.2254.

18. Cardoso VS, Magalhães AT, da Silva BAK, da Silva CS, Gomes DBC, Silva JCA. Avaliação funcional dos pés de portadores de diabetes tipo II. Rev Bras Promoc Saude. [Internet] 2013;26(4) [acesso em 10 jan 2017]. Disponível: http://www.unifor.br/images/pdfs/rbps/2013.4_artigo14.pdf.

19. Reis JS, Sociedade Brasileira de Diabetes (SBD). Cuide bem dos seus pés e não pise na bola! [Internet] 2011 [acesso em 10 jan 2017]. Disponível: http://www.diabetes.org.br/publico/colunas/39-dra-janice-sepulveda/172cuide-bem-dos-seus-pes-e-nao-pise-na-bola. 